\section{OAK RIDGE NATIONAL LABORATORY}

MARTIN MARIETTR
Coefficient Adaptive Triangulation for Strongly Anisotropic Problems

\author{
E. F. D'Azevedo \\ C. H. Romine \\ J. M. Donato
}

MANAGED BY

MARTIN MARIETTA ENERGY SYSTEMS, INC.

FOR THE UNITED STATES

DEPARTMENT OF ENERGY

DISTRISUTLN OF THS DOCUMERT IS UNLIATTED 
This report has been reproduced directly from the best available copy.

Available to DOE and DOE contractors from the Office of Scientific and Technical Information, P.O. Box 62, Oak Ridge, TN 37831; prices available from (615) 576-8401, FTS 626-8401.

Available to the public from the National Technical Information Service, U.S. Department of Commerce, 5285 Port Royal Rd., Springfield, VA 22161.

This report was prepared as an account of work sponsored by an agency of the United States Government. Neither the United States Government nor any agency thereof, nor any of their employees, makes any warranty, express or implied, or assumes any legal liability or responsibility for the accuracy, completeness, or usefulness of any information, apparatus, product, or process disclosed, or represents that its use would not infringe privately owned rights. Reference herein to any specific commercial product, process, or service by trade name, trademark, manufacturer, or otherwise, does not necessarily constifute or imply its endorsement, recommendation, or favoring by the United States Government or any agency thereof. The views and opinions of authors expressed herein do not necessarily state or reflect those of the United Stales Government or any agency thereof. 
Computer Science and Mathematics Division

Mathematical Sciences Section

\title{
COEFFICIENT ADAPTIVE TRIANGULATION FOR STRONGLY ANISOTROPIC PROBLEMS
}

\author{
E.F. D'Azevedo \\ C.H. Romine \\ J.M. Donato
}

Mathematical Sciences Section

Oak Ridge National Laboratory

P.O. Box 2008, Bldg. 6012

Oak Ridge, TN 37831-6367

Date Published: January 1996

Research supported by the Applied Mathematical Sciences subprogram of the Office of Energy Research, U.S. Department of Energy

Prepared by the

Oak Ridge National Laboratory

Oak Ridge, Tennessee 37831

managed by

Lockheed Martin Energy Research Corporation

for the

U.S. Department of Energy

under Contract No. DE-AC05-96OR22464 



\section{Contents}

1 Introduction . . . . . . . . . . . . . . . 1

2 Fourier Analysis of Anisotropic Problem . . . . . . . . . . 2

3 Coefficient Adaptive Triangulation $\ldots \ldots \ldots \ldots \ldots$

4 Stone's problem . . . . . . . . . . . . . . . . 6

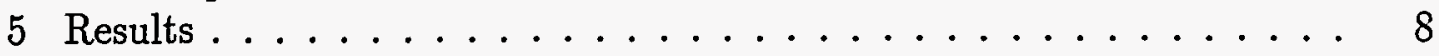

6 Summary . . . . . . . . . . . . . . . . . 11

7 References ............................ 12

- iii - 


\section{List of Figures}

4.1 Stone's third problem. . . . . . . . . . . . . . 7

4.2 Coefficient adaptive triangulation of Stone's Third Problem, $\gamma=100.7$

5.1 Accurate solution on $121 \times 121$ grid with $\gamma=100 \ldots \ldots \ldots 9$ 


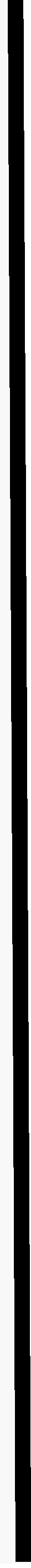




\title{
COEFFICIENT ADAPTIVE TRIANGULATION FOR STRONGLY ANISOTROPIC PROBLEMS
}

\author{
E.F. D'Azevedo \\ C.H. Romine \\ J.M. Donato
}

\begin{abstract}
Second order elliptic partial differential equations arise in many important applications, including flow through porous media, heat conduction, and the distribution of electrical or magnetic potential. The prototype is the Laplace problem, which in discrete form produces a coefficient matrix that is relatively easy to solve in a regular domain. However, the presence of anisotropy produces a matrix whose condition number is increased, making the resulting linear system more difficult to solve.

In this work, we take the anisotropy into account in the discretization by mapping each anisotropic region into a "stretched" coordinate space in which the anisotropy is removed. The region is then uniformly triangulated, and the resulting triangulation mapped back to the original space. The effect is to generate long slender triangles that are oriented in the direction of "preferred flow." Slender triangles are generally regarded as numerically undesirable since they tend to cause poor conditioning; however, our triangulation has the effect of producing effective isotropy, thus improving the condition number of the resulting coefficient matrix.
\end{abstract}

- vii - 



\section{Introduction}

Second order elliptic partial differential equations arise in many important applications, including flow through porous media, heat conduction, and the distribution of electrical potential. A simple prototype is the piecewise constant coefficient equation,

$$
\frac{\partial}{\partial x}\left(K_{x} \frac{\partial P}{\partial x}\right)+\frac{\partial}{\partial y}\left(K_{y} \frac{\partial P}{\partial y}\right)=-q
$$

which reduces to the Laplace problem for $K_{x}=K_{y}=1$. The discretization of the Laplace problem over a regular grid produces a coefficient matrix that is relatively easy to solve. However, the presence of strong anisotropy $\left(K_{x} \gg K_{y}\right)$ produces a poorly conditioned matrix, making the resulting linear system more difficult to solve.

In this report, we take the anisotropy into account in the discretization by mapping each anisotropic region into a "stretched" coordinate space in which the anisotropy is removed. The region is then uniformly triangulated, and the resulting triangulation mapped back to the original space. The effect is to generate long slender triangles that are oriented in the direction of "preferred flow." Slender triangles are generally regarded as numerically undesirable; however, our triangulation has the effect of producing effective isotropy, thus producing a coefficient matrix with a smaller condition number. Furthermore, our initial experiments suggest such "coefficient-adaptive" triangulation suffers no degradation in approximation accuracy.

The idea of using special approximation basis functions that depend on the rough coefficients has also been proposed by Falk and Osborn [3] in the analysis of mixed finite element methods for problems with rough coefficients. A technique of using Delaunay triangulation under an anisotropic transformation has been examined by Letniowski [9] and Forsyth [4]. The idea in their work is to ensure that the coefficient matrix resulting from the standard Galerkin finite element approximation of the second-order diffusion operator is an M-matrix. Our work 
differs in that our focus is on improving the conditioning of the linear system.

In Section 2, we describe the motivation for coefficient-adaptive mesh generation, using a simple example for illustration. In Section 3, we describe in more detail the approach that we have taken to discretizing anisotropic problems. Section 4 presents our sequence of test problems, based on Stone's third problem [10]. Section 5 presents the results of our empirical studies, comparing our coefficientadaptive discretization to the standard 5-point Laplacian discretization on the test problems. Finally, in Section 6, we summarize our conclusions and discuss how the results can be expanded.

\section{Fourier Analysis of Anisotropic Problem}

In this section, we use a Fourier analysis technique to analyze the condition of the coefficient matrix arising from the 5-point finite difference discretization of a model anisotropic problem. The analysis we present here follows the technique described by Chan and Elman [1] and Donato and Chan [2].

The problem we analyze is

$$
\frac{\partial}{\partial x}\left(K_{x} \frac{\partial P}{\partial x}\right)+\frac{\partial}{\partial y}\left(K_{y} \frac{\partial P}{\partial y}\right)=-q
$$

on the unit square with Neumann boundary conditions, where $K_{x}$ and $K_{y}$ are constant. Clearly, the differential equation with pure Neumann boundary conditions is not well-posed, and the resulting coefficient matrix will be rank-deficient. Rather than imposing an additional constraint, we define the modified condition number of the coefficient matrix to be

$$
\tilde{\kappa}=\frac{\lambda_{\max }}{\tilde{\lambda}_{\min }}
$$

where $\tilde{\lambda}_{\min }$ is the smallest nonzero eigenvalue of the matrix.

Let $h_{x}, h_{y}$ be the grid spacing in $x$ and $y$, so that $n_{x}=h_{x}^{-1}$ and $n_{y}=h_{y}^{-1}$ are the number of grid points in $x$ and $y$, respectively. We wish to compute the 
modified condition number as a function of $K_{x}, K_{y}, h_{x}$ and $h_{y}$. More accurately, we will compute the modified condition number as a function of the degree of anisotropy $\alpha=K_{y} / K_{x}$ and the grid aspect ratio $\rho=h_{y} / h_{x}$. The corresponding finite difference equations are:

$$
K_{x} \frac{-P_{i-1, j}+2 P_{i j}-P_{i+1, j}}{h_{x}^{2}}+K_{y} \frac{-P_{i, j-1}+2 P_{i j}-P_{i, j+1}}{h_{y}{ }^{2}}=q_{i j} .
$$

On the boundary of the square, the Neumann boundary conditions can be imposed using centered differences, yielding

$$
\begin{aligned}
& P_{i, j+1}=P_{i, j-1} \quad \text { for } i=0 \text { and } i=n_{x} \\
& P_{i+1, j}=P_{i-1, j} \quad \text { for } j=0 \text { and } j=n_{y} .
\end{aligned}
$$

If we scale (2.2) symmetrically so that the coefficient of $P_{i j}$ is 1 , we have

$$
P_{i j}+b\left(P_{i-1, j}+P_{i+1, j}\right)+c\left(P_{i, j-1}+P_{i, j+1}\right)=\tilde{q}_{i j}
$$

where $b=-\frac{1}{2}\left(1+\alpha / \rho^{2}\right)^{-1}$ and $c=-\frac{1}{2}\left(1+\rho^{2} / \alpha\right)^{-1}=-\frac{1}{2}-b$. A straightforward analysis of the spectrum of the difference operator leads to the following expression for the eigenvalues:

$$
\lambda_{i j}=1+2 b \cos \theta_{i}+2 c \cos \phi_{j}
$$

where $\theta_{i}=i \pi h_{x}$ for $i=0, \ldots, n_{x}-1$ and $\phi_{j}=j \pi h_{y}$ for $j=0, \ldots, n_{y}-1$.

The pure Neumann boundary conditions mean that $(1,1, \ldots, 1)^{T}$ is an eigenvector, with corresponding eigenvalue $\lambda_{00}=0$. Furthermore, since $b$ and $c$ are both negative, $\lambda_{\max }$ occurs at $i=n_{x}-1$ and $j=n_{y}-1$, yielding $\lambda_{\max }=2$. By inspection, $\tilde{\lambda}_{\min }=\min \left\{\lambda_{10}, \lambda_{01}\right\}$. To determine which of these eigenvalues is smaller, we use the Taylor approximation $\cos \theta=1-\theta^{2} / 2+O\left(\theta^{4}\right)$. This yields

$$
\lambda_{10}=\frac{1}{2}\left(1+\alpha / \rho^{2}\right)^{-1}\left(\pi^{2} h_{x}^{2}\right)+O\left(h_{x}^{4}\right)
$$




$$
\lambda_{01}=\frac{1}{2}\left(1+\rho^{2} / \alpha\right)^{-1}\left(\pi^{2} h_{y}^{2}\right)+O\left(h_{y}^{4}\right)
$$

If we ignore the fourth-order terms, simple algebraic manipulation verifies that $\lambda_{01}=\alpha \lambda_{10}$. Hence, if $K_{x}>K_{y}$ so that $\alpha<1$, then $\tilde{\lambda}_{\min }=\lambda_{01}$. Conversely, if $K_{x}<K_{y}, \tilde{\lambda}_{\min }=\lambda_{10}$.

We now have an expression for the modified condition number, $\tilde{\kappa}=\lambda_{\max } / \tilde{\lambda}_{\min }$ as a function of $\alpha$ and $\rho$. If $K_{y}>K_{x}$, then

$$
\tilde{\kappa}(\alpha, \rho) \approx \frac{4\left(1+\alpha / \rho^{2}\right)}{\pi^{2} h_{x}^{2}}
$$

If $K_{y}<K_{x}$, then

$$
\tilde{\kappa}(\alpha, \rho) \approx \frac{4\left(1+\rho^{2} / \alpha\right)}{\pi^{2} h_{y}^{2}}
$$

Now we have the machinery in place to answer our primary question concerning the construction of a discretization grid: For a given degree of anisotropy (fixed $\alpha$ ) and a given number of unknowns $N$, what is the grid aspect ratio ( $\rho$ ) that minimizes the (modified) condition number of the resulting coefficient matrix? If we assume that $\alpha>1$, then we seek to minimize

$$
\tilde{\kappa} \approx \frac{4\left(1+\alpha / \rho^{2}\right)}{\pi^{2} h_{x}^{2}} .
$$

Using the fact that $N=n_{x} n_{y}=h_{x}^{-1} h_{y}^{-1}$ we can rewrite this as

$$
\tilde{\kappa}=4 \pi^{2} N(\rho+\alpha / \rho)
$$

for which the minimum occurs at $\rho=\sqrt{\alpha}$. The same result is obtained for $\alpha<1$. We use this result in defining the mapping described in Section 3. The smallest condition number is obtained when $\alpha=1$, indicating that the isotropic case produces the best conditioned matrix.

To summarize, for $\rho=\sqrt{\alpha}$, we have

$$
\tilde{\kappa} \approx \frac{8 N}{\pi^{2}}\left(\max \left\{K_{y} / K_{x}, K_{x} / K_{y}\right\}\right)^{1 / 2}
$$


We used a series of MATLAB tests to verify the conclusions of the analysis described above. Table 2.1 is in three sections. The first four rows display the close agreement between the condition number $\tilde{\kappa}_{F D M}$ of the coefficient matrix and the theoretical condition number $\tilde{\kappa}$, both computed by MATLAB. Two digits of agreement are obtained even for very small problems.

The second section of the table verifies that as the degree of anisotropy $(\alpha)$ is multiplied (or divided) by 100 , the optimal condition number $\tilde{\kappa}$ increases by $\sqrt{100}=10$, as predicted. The second and third sections of the table together illustrate that choosing the correct aspect ratio $\rho$ can significantly improve the condition number of the resulting coefficient matrix.

\begin{tabular}{|r|r|r|r|r|r|r|}
\hline$\alpha$ & $n_{x}$ & $n_{y}$ & $n_{x} * n_{y}$ & \# unknowns & $\tilde{\kappa}$ & $\tilde{\kappa}_{F D M}$ \\
\hline 1 & 12 & 12 & 144 & 169 & $1.1672 \mathrm{e}+02$ & $1.1739 \mathrm{e}+02$ \\
\hline 2 & 14 & 10 & 140 & 165 & $1.6049 \mathrm{e}+02$ & $1.6117 \mathrm{e}+02$ \\
\hline 4 & 16 & 8 & 128 & 153 & $2.0751 \mathrm{e}+02$ & $2.0817 \mathrm{e}+02$ \\
\hline 10 & 21 & 6 & 126 & 154 & $3.2463 \mathrm{e}+02$ & $3.2524 \mathrm{e}+02$ \\
\hline \hline 5 & 5,981 & 2,674 & $15,993,194$ & $16,001,850$ & $2.8987 \mathrm{e}+07$ & - \\
\hline 500 & 18,914 & 845 & $15,982,330$ & $16,002,090$ & $2.8968 \mathrm{e}+08$ & - \\
\hline $1 / 500$ & 845 & 18,914 & $15,982,330$ & $16,002,090$ & $2.8968 \mathrm{e}+08$ & - \\
\hline \hline 5 & 4,000 & 4,000 & $16,000,000$ & $16,008,001$ & $3.8907 \mathrm{e}+07$ & - \\
\hline 500 & 4,000 & 4,000 & $16,000,000$ & $16,008,001$ & $3.2488 \mathrm{e}+09$ & - \\
\hline $1 / 500$ & 4,000 & 4,000 & $16,000,000$ & $16,008,001$ & $3.2488 \mathrm{e}+09$ & - \\
\hline
\end{tabular}

Table 2.1: MATLAB results verifying the analysis.

\section{Coefficient Adaptive Triangulation}

We modified an existing triangular mesh generation package, GEOMPACK [7], to generate coefficient adaptive triangulations. GEOMPACK is a mathematical software package written in Fortran 77 for the generation of convex polygon decompositions and triangular meshes in two-dimensional polygonal regions.

GEOMPACK generates a triangular mesh by first decomposing the polygonal domain into simpler convex polygons [6]. The decomposition can be further 
controlled by equidistribution of a user supplied density function. We chose to use $\left(K_{x} K_{y}\right)^{1 / 2}$ as the density function, to allocate proportionally more triangles in regions where $K_{x}$ or $K_{y}$ is small. GEOMPACK then generates a uniform triangulation within the interior of each convex subdomain [5]. A final step handles the mesh connection between neighboring subdomains [8] to generate a Delaunay triangulation.

GEOMPACK was modified to perform a rescaling by $K_{x}^{-1 / 2}$ and $K_{y}^{-1 / 2}$ in the $x$ and $y$ directions before the generation of a uniform mesh within each convex subdomain. The node coordinates of this triangulation are then mapped back into the original space.

\section{Stone's problem}

We tested the technique for coefficient adaptive triangulation on a variant of Stone's third problem [10] in solving

$$
\frac{\partial}{\partial x}\left(K_{x} \frac{\partial P}{\partial x}\right)+\frac{\partial}{\partial y}\left(K_{y} \frac{\partial P}{\partial y}\right)=-q
$$

on the $[0,30] \times[0,30]$ with Neumann boundary conditions. Locations and strengths of point sources and sinks are

$$
\begin{array}{r}
q_{1}(3,3)=1.0, \quad q_{2}(3,37)=0.5, \quad q_{3}(23,4)=0.6 \\
q_{4}(14,15)=-1.83, \quad q 5(27,27)=-0.27
\end{array}
$$

The distribution of material properties, $K_{x}, K_{y}$ were (see Figure 4.1)

$$
\left(K_{x}, K_{y}\right)=\left\{\begin{array}{lll}
(1, \gamma) & \text { if }\left(x_{i}, y_{j}\right) \in B, \quad 14 \leq i \leq 30,0 \leq j \leq 16 \\
(\gamma, 1) & \text { if }\left(x_{i}, y_{j}\right) \in C, \quad 5 \leq i \leq 12,5 \leq j \leq 12 \\
(0,0) & \text { if }\left(x_{i}, y_{j}\right) \in D, \quad 12 \leq i \leq 19,21 \leq j \leq 28 \\
(1,1) & \text { if }\left(x_{i}, y_{j}\right) \in A .
\end{array}\right.
$$

A $31 \times 31$ regular grid with $\gamma=100$ was used in the original problem. Note 


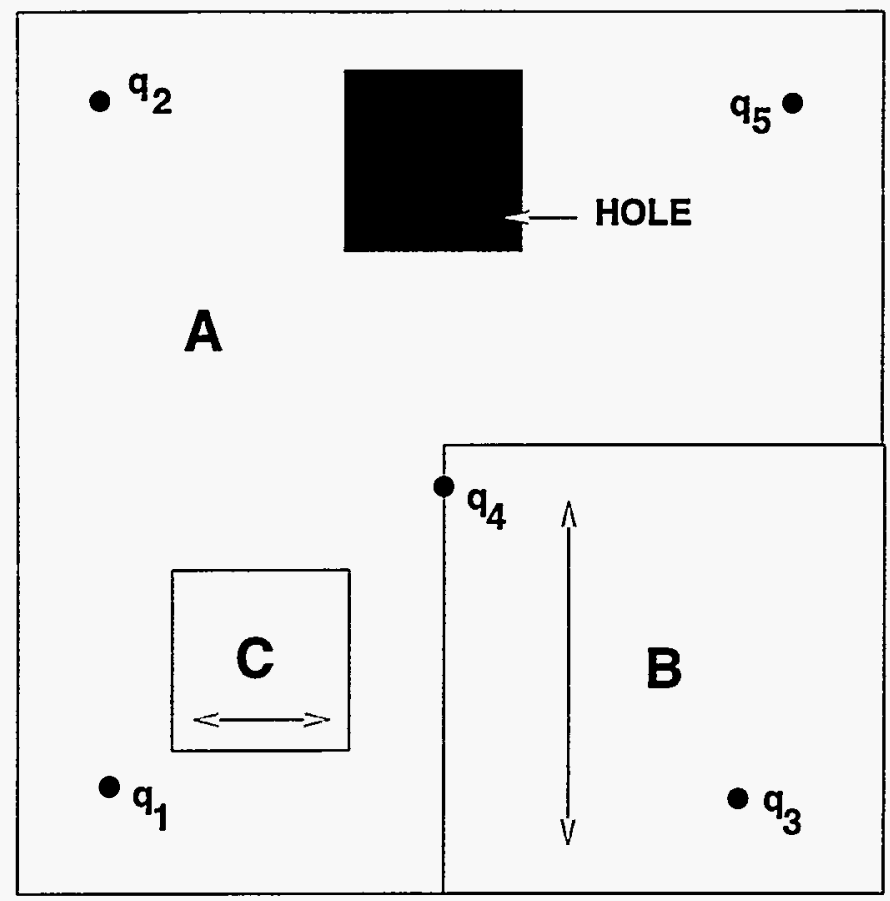

Figure 4.1: Stone's third problem.

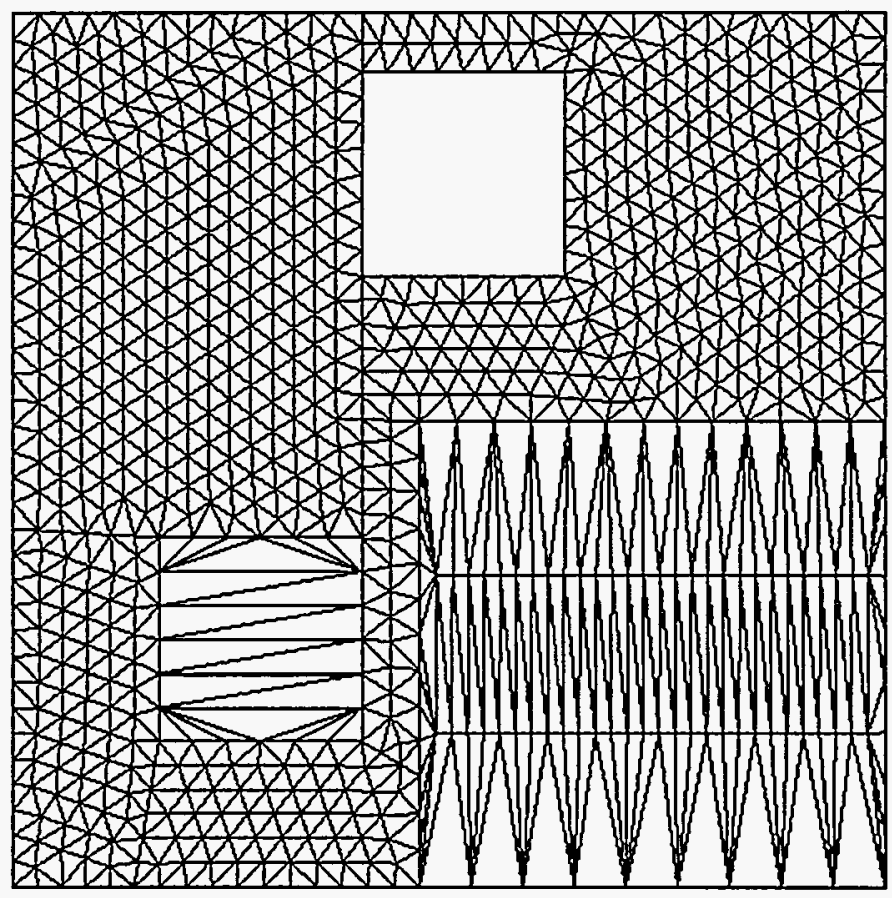

Figure 4.2: Coefficient adaptive triangulation of Stone's Third Problem, $\gamma=100$. 
that region $D$ with $K_{x}=K_{y}=0$ was modeled as a hole in the plate. We chose a consistent discretization scheme based on linear triangular elements in a Galerkin Finite Element formulation. Even on a regular rectangular grid, we generate a triangulation by consistently splitting each rectangle into two rightangle triangles. For the regular rectangular grid, this yields the identical standard 5 -point finite difference stencil, with the exception of the nodes at interfaces between different materials.

\section{Results}

We chose MATLAB to perform the numerical experiments and visualization. We used linear triangular elements in a Galerkin Finite Element formulation to perform the matrix assembly. Initially, we imposed a Dirichlet condition at the origin $(0,0)$ to avoid exact rank deficiency. However, subsequent testing revealed that the CG iteration applied to the original semi-definite problem converged more rapidly than CG iteration on the problem with a Dirichlet condition imposed. We have reported the times for both problems in the interest of completeness. To simplify visualization, all solutions were interpolated and compared on a $31 \times 31$ regular rectangular mesh. Since region $D$ is modeled as a hole, nodes within region $D$ were set to zero for simplicity. We used the solution obtained from a $121 \times 121$ grid as an accurate solution. Note that the sources and sinks introduce point singularities; thus in comparing solution accuracies we ignore the errors within one mesh block of the point singularities.

Figure 4.2 displays the coefficient adaptive unstructured triangular mesh for $\gamma=100$. Note the orientation of slender triangles within regions $B$ and $C$. Figure 5.1 displays the accurate solution obtained with the $121 \times 121$ mesh for $\gamma=100$. Notice the flat solution profiles in regions $C$ and $D$ (see also Figure 4.1).

The linear systems resulting from the discretization were first scaled to unit diagonal, before they were solved using the conjugate gradient (CG) method with no further preconditioning. We used a relative reduction in the initial residual as 


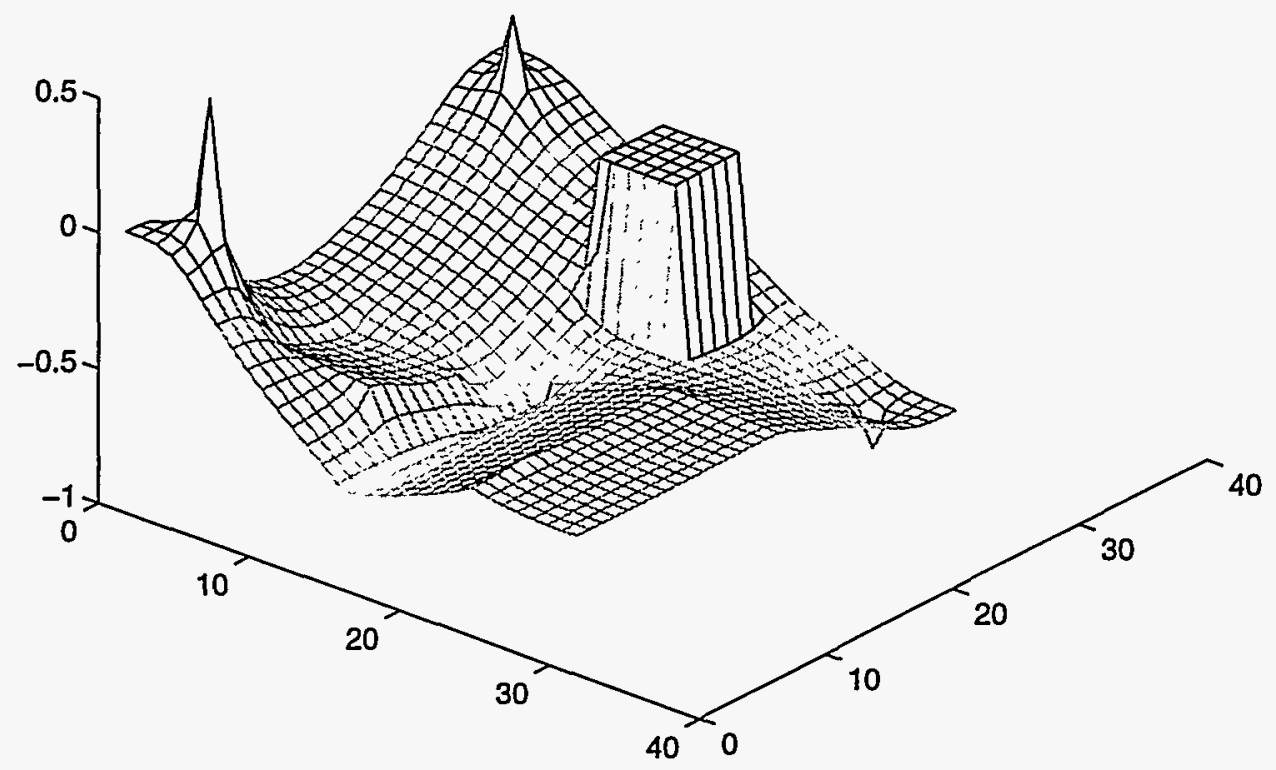

Figure 5.1: Accurate solution on $121 \times 121$ grid with $\gamma=100$.

our termination criterion:

$$
\left\|r_{k}\right\|_{2} \leq 10^{-10}\left\|r_{0}\right\|_{2}
$$

where $r_{k}$ is the residual vector on the $k$-th iteration. Tables 5.1 and 5.2 show the number of CG iterations, maximum discretization error and condition number. ${ }^{1}$

We also tested the problems using CG with SSOR preconditioning. If matrix $A=I-\left(L+L^{T}\right)$ represents the global assembled matrix with unit diagonal, and $L$ is strictly lower triangular, then the SSOR factorization used is

$$
Q=(I-\omega L)\left(I-\omega L^{T}\right)
$$

A few preliminary runs on various problem show the optimal $\omega$ to be between

\footnotetext{
${ }^{1}$ For the semidefinite case, we report the 'modified' condition number (2.1).
} 


\begin{tabular}{|r|ccc|ccc|cc|}
\hline & \multicolumn{3}{|c|}{ Regular Grid } & \multicolumn{4}{c|}{ Adaptive Triangulation } \\
\hline$\gamma$ & iters & err $^{a}$ & $\tilde{\kappa}$ & iters & err $^{a}$ & $\tilde{\kappa}$ & nelts $^{b}$ & $N^{c}$ \\
\hline 1 & 180 & $3.7 \mathrm{e}-2$ & $8.9 \mathrm{e}+2$ & 170 & $8.6 \mathrm{e}-3$ & $8.5 \mathrm{e}+2$ & 1653 & 900 \\
10 & 210 & $6.7 \mathrm{e}-2$ & $1.8 \mathrm{e}+3$ & 168 & $4.9 \mathrm{e}-2$ & $9.3 \mathrm{e}+2$ & 1635 & 894 \\
100 & 367 & $8.4 \mathrm{e}-2$ & $1.3 \mathrm{e}+4$ & 202 & $3.2 \mathrm{e}-2$ & $1.7 \mathrm{e}+3$ & 1589 & 870 \\
1000 & 629 & $7.7 \mathrm{e}-2$ & $1.2 \mathrm{e}+5$ & 278 & $1.7 \mathrm{e}-2$ & $8.9 \mathrm{e}+3$ & 1579 & 865 \\
\hline
\end{tabular}

Table 5.1: Diagonally Scaled PCG on Stone's Third Problem, No Dirichlet Boundary Condition Imposed

\begin{tabular}{|r|cc|cc|cc|}
\hline & \multicolumn{3}{|c|}{ Regular Grid } & \multicolumn{4}{|c|}{ Adaptive Triangulation } \\
\hline$\gamma$ & iters & $\kappa$ & iters & $\kappa$ & nelts $^{b}$ & $N^{c}$ \\
\hline 1 & 207 & $1.9 \mathrm{e}+4$ & 195 & $1.8 \mathrm{e}+4$ & 1653 & 900 \\
10 & 243 & $5.0 \mathrm{e}+4$ & 190 & $2.1 \mathrm{e}+4$ & 1635 & 894 \\
100 & 418 & $3.6 \mathrm{e}+5$ & 227 & $4.5 \mathrm{e}+4$ & 1589 & 870 \\
1000 & 665 & $3.4 \mathrm{e}+6$ & 305 & $2.2 \mathrm{e}+5$ & 1579 & 865 \\
\hline
\end{tabular}

Table 5.2: Diagonally Scaled PCG on Stone's Third Problem, Dirichlet Boundary Condition Imposed

${ }^{a}$ Maximum discretization error, estimated by comparing a highly accurate solution of the resulting linear system to the 'exact' solution.

${ }^{b}$ Number of triangular elements in the discretization.

${ }^{c}$ Total number of unknowns in the resulting linear system. 
1.4 and 1.6. A value of $\omega=1.5$ was consistently used for all runs for simplicity. All matrices were also consistently reordered using the bandwidth reducing RCM (Reverse Cuthill-McKee) ordering to minimize the effect of matrix ordering on convergence. The results are summarized in Table 5.3.

\begin{tabular}{|r|c|c|}
\hline & Regular Grid & Adaptive Triangulation \\
\hline$\gamma$ & iters & iters \\
1 & 47 & 51 \\
10 & 56 & 53 \\
100 & 104 & 70 \\
1000 & 207 & 111 \\
\hline
\end{tabular}

Table 5.3: Results of SSOR PCG on Stone's Third Problem

The results from Tables 5.1 and 5.3 show coefficient adaptive triangulations generate better conditioned matrices with no loss of approximation accuracy even using slender triangles.

\section{Summary}

We have explored the use of coefficient adaptive mesh generation techniques on strongly anisotropic problems. The initial results on Stone's problem suggest there is no loss in approximation accuracy even with slender triangles and the resulting discretization produces better conditioned matrices.

More extensive testing with more realistic problems is required. It is straightforward to extend this approach to generating tetrahedral meshes in three-dimensions.

\section{Acknowledgments}

The authors thank Professor Barry Joe for providing GEOMPACK. We also thank Pat Worley and Steve Lee for their insightful comments, which improved the paper. 
1

$\checkmark$ 
ORNL/TM-13086

\section{INTERNAL DISTRIBUTION}

1. B. R. Appleton

2. B. A. Carreras

3-7. E. F. D'Azevedo

8. T. S. Darland

9-13. J. M. Donato

14. J. J. Dongarra

15. J. B. Drake

16. T. H. Dunigan

17. G. A. Geist

18. K. L. Kliewer

19-23. M. R. Leuze

24. E. G. $\mathrm{Ng}$

25. C. E. Oliver

26. B. W. Peyton

27-31. S. A. Raby
32. B. A. Riley

33-37. C. H. Romine

38. W. A. Shelton

39-43. R. F. Sincovec

44. G. M. Stocks

45. M. R. Strayer

46. D. W. Walker

47. P. H. Worley

48. T. Zacharia

49. Central Research Library

50. ORNL Patent Office

51. K-25 Applied Technology Library

52. Y-12 Technical Library

53. Laboratory Records - RC

54-55. Laboratory Records Dept.

\section{EXTERNAL DISTRIBUTION}

56. Loyce M. Adams, Applied Mathematics, FS-20, University of Washington, Seattle, WA 98195

57. Christopher R. Anderson, Department of Mathematics, University of California, Los Angeles, CA 90024

58. Todd Arbogast, Department of Mathematical Sciences, Rice University, P. O. Box 1892, Houston, TX 77251

59. Donald M. Austin, 6196 EECS Building, University of Minnesota, 200 Union Street, S.E., Minneapolis, MN 55455

60. Robert G. Babb, Oregon Graduate Center, CSE Department, 19600 N.W. Walker Road, Beaverton, OR 97006

61. David H. Bailey, NASA Ames, Mail Stop 258-5, NASA Ames Research Center, Moffet Field, CA 94035

62. Jesse L. Barlow, Department of Computer Science and Engineering, 220 Pond Laboratory, The Pennsylvania State University, University Park, PA 16802-6106

63. Edward H. Barsis, Computer Science and Mathematics, P. O. Box 5800, Sandia National Laboratory, Albuquerque, NM 87185

64. Adam Beguelin, Carnegie Mellon University, School of Computer Science, 5000 Forbes Avenue, Pittsburgh, PA 15213-3890

65. Robert E. Benner, Parallel Processing Division 1413, Sandia National Laboratories, P. O. Box 5800, Albuquerque, NM 87185 
66. Marsha J. Berger, Courant Institute of Mathematical Sciences, 251 Mercer Street, New York, NY 10012

67. Philippe Berger, Institut National Polytechnique, ENSEEIHT, 2 rue Charles CamichelF, 31071 Toulouse Cedex, France

68. Ake Bjorck, Department of Mathematics, Linkoping University, S-581 83 Linkoping, Sweden

69. John H. Bolstad, L-16, Lawrence Livermore National Laboratory, P. O. Box 808, Livermore, CA 94550

70. Roger W. Brockett, Harvard University, Pierce Hall, 29 Oxford Street Cambridge, MA 02138

71. James C. Browne, Department of Computer Sciences, University of Texas, Austin, TX 78712

72. Bill L. Buzbee, Scientific Computing Division, National Center for Atmospheric Research, P. O. Box 3000, Boulder, CO 80307

73. Donald A. Calahan, Department of Electrical and Computer Engineering, University of Michigan, Ann Arbor, MI 48109

74. Thomas A. Callcott, Director, The Science Alliance Program, 53 Turner House, University of Tennessee, Knoxville, TN 37996

75. Tony Chan, Department of Mathematics, University of California, Los Angeles, 405 Hilgard Avenue, Los Angeles, CA 90024

76. Jagdish Chandra, Army Research Office, P. O. Box 12211, Research Triangle Park, NC 27709

77. Siddhartha Chatterjee, Dept. of Computer Science, CB 3175, Sitterson Hall, The University of North Carolina, Chapel Hill, NC 27599-3175

78. Melvyn Ciment, National Science Foundation, 1800 G Street N.W., Washington, DC 20550

79. Tom Coleman, Department of Computer Science, Cornell University, Ithaca, NY 14853

80. Alva Couch, Department of Computer Science, Tufts University, Medford, MA 02155

81. Paul Concus, Mathematics and Computing, Lawrence Berkeley Laboratory, Berkeley, CA 94720

82. Tom Crockett, ICASE, Mail Stop 132C, NASA Langley Research Center, Hampton, VA 23665-5225

83. Jane K. Cullum, IBM T. J. Watson Research Center, P. O. Box 218, Yorktown Heights, NY 10598

84. George Cybenko, Center for Supercomputing Research and Development, University of Illinois, 104 South Wright Street, Urbana, IL 61801-2932

85. Helen Davis, Computer Science Department, Stanford University, Stanford, CA 94305

86. Michel Dayde, Institut National Polytechnique, ENSEEIHT, 2 rue Charles CamichelF, 31071 Toulouse Cedex, France 
87. Craig Douglas, IBM T. J. Watson Research Center, P. O. Box 218, Yorktown Heights, NY 10598-0218

88. Iain S. Duff, Atlas Centre, Rutherford Appleton Laboratory, Chilton, Oxon OX11 OQX, England

89. Victor Eijkhout, 405 Hilgard Avenue, Department of Mathematics, UCLA, Los Angeles, CA 90024

90. Stanley Eisenstat, Department of Computer Science, Yale University, P. O. Box 2158 Yale Station, New Haven, CT 06520

91. Howard C. Elman, Computer Science Department, University of Maryland, College Park, MD 20742

92. Richard E. Ewing, Director, Institute for Scientific Computations, Texas A\&M University, College Station, TX 77843-3404

93. Edward Felten, Department of Computer Science, University of Washington, Seattle, WA 98195

94. Charles Fineman, Ames Research Center, Mail Stop 269/3, Moffet Field, CA 94035

95. David Fisher, Department of Mathematics, Harvey Mudd College, Claremont, CA 91711

96. Jon Flower, Parasoft Corporation, 2500 E. Foothill Boulevard, Suite 205, Pasadena, CA 91107

97. Geoffrey C. Fox, NPAC, 111 College Place, Syracuse University, Syracuse, NY $13244-4100$

98. Chris Fraley, Statistical Sciences, Inc., 1700 Westlake Avenue N, Suite 500, Seattle, WA 98119

99. Joan M. Francioni, Computer Science Department, University of Southwestern Louisiana, Lafayette, LA 70504

100. Paul O. Frederickson, ACL, MS B287, Los Alamos National Laboratory, Los Alamos, NM 87545

101. Offir Frieder, George Mason University, Science and Technology Building, Computer Science Department, 4400 University Drive, Fairfax, Va 22030-4444

102. Robert E. Funderlic, Department of Computer Science, North Carolina State University, Raleigh, NC 27650

103. Dennis B. Gannon, Computer Science Department, Indiana University, Bloomington, IN 47401

104. C. William Gear, NEC Research Institute, 4 Independence Way, Princeton, NJ 08540

105. W. Morven Gentleman, Division of Electrical Engineering, National Research Council, Building M-50, Room 344, Montreal Road, Ottawa, Ontario, Canada K1A 0R8

106. Alan George, Vice President, Academic and Provost, Needles Hall, University of Waterloo, Waterloo, Ontario, Canada N2L 3G1 
107. James Glimm, SUNY-Stony Brook, Department of Applied Mathematics and Statistics, Stony Brook, NY 11794

108. Gene Golub, Computer Science Department, Stanford University, Stanford, CA 94305

109. Joseph F. Grcar, Division 8245 , Sandia National Laboratories, Livermore, CA 94551-0969

110. William D. Gropp, Mathematics and Computer Science Division, Argonne National Laboratory, 9700 South Cass Avenue, Argonne, IL 60439

111. Eric Grosse, AT\&T Bell Labs 2T-504, Murray Hill, NJ 07974

112. Sanjay Gupta, ICASE, Mail Stop 132C, NASA Langley Research Center, Hampton, VA 23665-5225

113. John L. Gustafson, Ames Laboratory, 236 Wilhelm Hall, Iowa State University, Ames, IA 50011-3020

114. Christian Halloy, Assistant Director of JICS, 104 South College, Joint Institute for Computational Science, University of Tennessee, Knoxville, TN 37996-1301

115. Sven J. Hammarling, The Numerical Algorithms Group, Ltd., Wilkinson House, Jordan Hill Road, Oxford OX2 8DR, United Kingdom

116. Robert M. Haralick, Department of Electrical Engineering, Director, Intelligent Systems Lab, University of Washington, 402 Electrical Engineering Building, FT10, Seattle, WA 98195

117. Ann H. Hayes, Computing and Communications Division, Los Alamos National Laboratory, Los Alamos, NM 87545

118. Michael T. Heath, National Center for Supercomputing Applications, 4157 Beckman Institute University of Illinois, 405 North Mathews Avenue, Urbana, IL 61801-2300

119. Gerald W. Hedstrom, L-71, Lawrence Livermore National Laboratory, P. O. Box 808, Livermore, CA 94550

120. Don E. Heller, Ames Laboratory, 327 Wilhelm, Ames, IA 50011

121. John L. Hennessy, CIS 208, Stanford University, Stanford, CA 94305

122. N. J. Higham, Department of Mathematics, University of Manchester, Gtr Manchester, M13 9PL, England

123. Dan Hitchcock, Office of Scientific Computing, ER-7, Applied Mathematical Sciences, Office of Energy Research, U.S. Department of Energy, Washington, DC 20585

124. Charles J. Holland, Air Force Office of Scientific Research, Building 410, Bolling Air Force Base, Washington, DC 20332

125. Fred Howes, Office of Scientific Computing, ER-7, Applied Mathematical Sciences, Office of Energy Research, Department of Energy, Washington, DC 20585

126. Robert E. Huddleston, Computation Department, Lawrence Livermore National Laboratory, P. O. Box 808, Livermore, CA 94550

127. Jenq-Neng Hwang, Department of Electrical Engineering, FT-10, University of Washington, Seattle, WA 98195 
128. Ilse Ipsen, Department of Computer Science, Yale University, P. O. Box 2158 Yale Station, New Haven, CT 06520

129. Leah H. Jamieson, School of Electrical Engineering, Purdue University, West Lafayette, IN 47907

130. Gary Johnson, Office of Scientific Computing, ER-7, Applied Mathematical Sciences, Office of Energy Research, U.S. Department of Energy, Washington, DC 20585

131. Lennart Johnsson, Thinking Machines Corporation, 245 First Street, Cambridge, MA 02142-1214

132. Harry Jordan, Department of Electrical and Computer Engineering, University of Colorado, Boulder, CO 80309

133. Bo Kagstrom, Institute of Information Processing, University of Umea, 5-901 87 Umea, Sweden

134. Malvyn Kalos, Cornell Theory Center, Engineering and Theory Center Building, Cornell University, Ithaca, NY 14853-3901

135. Hans Kaper, Mathematics and Computer Science Division, Argonne National Laboratory, 9700 South Cass Avenue, BIdg. 221, Argonne, IL 60439

136. Alan H. Karp, HP Labs 3U-7, Hewlett-Packard Company, 1501 Page Mill Road, Palo Alto, CA 94304

137. Linda Kaufman, Bell Laboratories, 600 Mountain Avenue, Murray Hill, NJ 07974

138. Robert J. Kee, Applied Mathematics Division 8245, Sandia National Laboratories, Livermore, CA 94551-0969

139. Kenneth Kennedy, Department of Computer Science, Rice University, P.O. Box 1892, Houston, TX 77001

140. Tom Kitchens, Office of Scientific Computing, ER-7, Applied Mathematical Sciences, Office of Energy Research, U.S. Department of Energy, Washington, DC 20585

141. Clyde P. Kruskal, Department of Computer Science, University of Maryland, College Park, MD 20742

142. Edward Kushner, Intel Corporation, 15201 NW Greenbrier Parkway, Beaverton, OR 97006

143. Michael Langston, Department of Computer Science, University of Tennessee, Knoxville, TN 37996-1301

144. Richard Lau, Office of Naval Research, Code 111MA 800 Quincy Street, Boston Tower 1, Arlington, VA 22217-5000

145. Robert L. Launer, Army Research Office, P. O. Box 12211, Research Triangle Park, NC 27709

146. Tom Leighton, Lab for Computer Science, Massachusetts Institute of Technology, 545 Technology Square, Cambridge, MA 02139

147. Robert Leland, Sandia National Laboratories, 1424, P. O. Box 5800, Albuquerque, NM 87185-5800 
148. Randall J. LeVeque, Applied Mathematics, FS-20, University of Washington, Seattle, WA 98195

149. John G. Lewis, Boeing Computer Services, P. O. Box 24346, M/S 7L-21, Seattle, WA 98124-0346

150. Heather M. Liddell, Center for Parallel Computing, Department of Computer Science and Statistics, Queen Mary College, University of London, Mile End Road, London E1 4NS, England

151. Brent Lindquist, SUNY-Stony Brook, Department of Applied Mathematics and Statistics, Stony Brook, NY 11794

152. Rik Littlefield, Pacific Northwest Laboratory, MS K1-87, P.O.Box 999, Richland, WA 99352

153. Joseph Liu, Department of Computer Science, York University, 4700 Keele Street, Downsview, Ontario, Canada M3J 1P3

154. Franklin Luk, Department of Computer Science, Amos Eaton Building - No. 131 Rensselaer Polytechnic Institute Troy, NY 12180-3590

155. Ewing Lusk, Mathematics and Computer Science Division, Argonne National Laboratory, 9700 South Cass Avenue, MCS 221 Argonne, IL 60439-4844

156. Allen D. Malony, Department of Computer and Information Science, University of Oregon, Eugene, OR 97403

157. Thomas A. Manteuffel, Department of Mathematics, University of Colorado Denver, Denver, CO 80202

158. Anita Mayo, IBM T. J. Watson Research Center, P. O. Box 218, Yorktown Heights, NY 10598

159. Oliver McBryan, University of Colorado at Boulder, Department of Computer Science, Campus Box 425, Boulder, CO 80309-0425

160. James McGraw, Lawrence Livermore National Laboratory, L-306, P. O. Box 808, Livermore, CA 94550

161. Piyush Mehrotra, ICASE, Mail Stop 132C, NASA Langley Research Center, Hampton, VA 23665

162. Paul C. Messina, Mail Code 158-79, California Institute of Technology, $1201 \mathrm{E}$. California Boulevard, Pasadena, CA 91125

163. Cleve B. Moler, MathWorks, 325 Linfield Place, Menlo Park, CA 94025

164. Jorge J. More, Mathematics and Computer Science Division, Argonne National Laboratory, 9700 South Cass Avenue, Argonne, IL 60439

165. William A. Mulder, Koninklijke Shell Exploratie en Produktie Laboratorium, Postbus 60, 2280 AB Rijswijk, The Netherlands

166. David Nelson, Director, Office of Scientific Computing, ER-7, Applied Mathematical Sciences, Office of Energy Research, U.S. Department of Energy, Washington, DC 20585

167. V. E. Oberacker, Department of Physics, Vanderbilt University, Box 1807, Station B, Nashville, TN 37235 
168. Dianne P. O'Leary, Computer Science Department, University of Maryland, College Park, MD 20742

169. Joseph Oliger, Computer Science Department, Stanford University, Stanford, CA 94305

170. James M. Ortega, Department of Computer Science, Thornton Hall, University of Virginia, Charlottesville, VA 22901

171. Steve Otto, Oregon Graduate Institute, Department of Computer Science and Engineering, 19600 NW von Neumann Drive, Beaverton, OR 97006-1999

172. Cherri Pancake, Department of Computer Science, Oregon State University, Corvallis, OR 97331-3202

173. Joseph E. Pasciak, Applied Mathematics, Brookhaven National Laboratory, Upton, NY 11973

174. Merrell Patrick, National Science Foundation, 1800 G Street N.W., Washington, DC 20550

175. David Payne, Intel Corporation, Supercomputer Systems Division, $15201 \mathrm{NW}$ Greenbrier Parkway, Beaverton, OR 97006

176. Ronald F. Peierls, DAS - Bldg. 490-D, P.O. Box 5000, Brookhaven National Laboratory, Upton, NY 11973

177. Linda R. Petzold, Computer Science Department, University of Minnesota, 200 Union Street, S.E., Room 4-192, Minneapolis, MN 55455

178. Dan Pierce, Boeing Computer Services, P.O. Box 24346, M/S 7L-21, Seattle, WA 98124-0346

179. Paul Pierce, Intel Corporation, Supercomputer Systems Division, 15201 NW Greenbrier Parkway, Beaverton, OR 97006

180. Robert J. Plemmons, Departments of Mathematics and Computer Science, Box 7311, Wake Forest University, Winston-Salem, NC 27109

181. James C. T. Pool, Deputy Director, Caltech Concurrent Supercomputing Facility, California Institute of Technology, MS 158-79, Pasadena, CA 91125

182. Jesse Poore, Computer Science Department, University of Tennessee, Knoxville, TN 37996-1300

183. David A. Poplawski, Department of Computer Science, Michigan Technological University, Houghton, MI 49931

184. Roldan Pozo, University of Tennessee, 107 Ayres Hall, Department of Computer Science, Knoxville, TN 37996-1301

185. Padma Raghavan, University of Illinois, NCSA, 4151 Beckman Institute, 405 North Matthews Avenue, Urbana, IL 61801

186. Daniel A. Reed, Computer Science Department, University of Illinois, Urbana, IL 61801

187. John K. Reid, Numerical Analysis Group, Central Computing Department, Atlas Centre, Rutherford Appleton Laboratory, Didcot, Oxon OX11 0QX, England

188. John R. Rice, Computer Science Department, Purdue University, West Lafayette, IN 47907 
189. Garry Rodrigue, Numerical Mathematics Group, Lawrence Livermore National Laboratory, Livermore, CA 94550

190. Donald J. Rose, Department of Computer Science, Duke University, Durham, NC 27706

191. Ahmed H. Sameh, Department of Computer Science, University of Minnesota, 200 Union Street S.E., Minneapolis, MN 55455

192. Joel Saltz, Computer Science Department, A.V. Williams Building, University of Maryland, College Park, MD 20742

193. Jorge Sanz, IBM Almaden Research Center, Department K53/802, 650 Harry Road, San Jose, CA 95120

194. Robert B. Schnabel, Department of Computer Science, University of Colorado at Boulder, ECOT 7-7 Engineering Center, Campus Box 430, Boulder, CO 803090430

195. Robert Schreiber, RIACS, MS T27A-1, NASA Ames Research Center, Moffet Field, CA 94035

196. Martin H. Schultz, Department of Computer Science, Yale University, P. O. Box 2158 Yale Station, New Haven, CT 06520

197. David S. Scott, Intel Scientific Computers, 15201 N.W. Greenbrier Parkway, Beaverton, OR 97006

198. The Secretary, Department of Computer Science and Statistics, The University of Rhode Island, Kingston, RI 02881

199. Charles L. Seitz, Department of Computer Science, California Institute of Technology, Pasadena, CA 91125

200. Margaret L. Simmons, Computing and Communications Division, Los Alamos National Laboratory, Los Alamos, NM 87545

201. Horst D. Simon, NASA Ames Research Center, Mail Stop T045-1, Moffett Field, CA 94035

202. William C. Skamarock, 3973 Escuela Court, Boulder, CO 80301

203. Tony Skjellum, Dept of Computer Science, Mississippi State University, PO Drawer CS, Mississippi State, MS 39762-5623

204. Burton Smith, Tera Computer Company, 400 North 34th Street, Suite 300, Seattle, WA 98103

205. Marc Snir, IBM T.J. Watson Research Center, Department 420/36-241, P. O. Box 218, Yorktown Heights, NY 10598

206. Larry Snyder, Department of Computer Science and Engineering, FR-35, University of Washington, Seattle, WA 98195

207. Danny C. Sorensen, Department of Mathematical Sciences, Rice University, P. O. Box 1892, Houston, TX 77251

208. Rick Stevens, Mathematics and Computer Science Division, Argonne National Laboratory, 9700 South Cass Avenue, Argonne, IL 60439

209. G. W. Stewart, Computer Science Department, University of Maryland, College Park, MD 20742 
210. Paul N. Swarztrauber, National Center for Atmospheric Research, P. O. Box 3000, Boulder, CO 80307

211. Julie Swisshelm, Sandia National Laboratories, 1421, Parallel Computational Sciences Department, Albuquerque, New Mexico 87185-5800

212. Wei Pai Tang, Department of Computer Science, University of Waterloo, Waterloo, Ontario, Canada N2l 3G1

213. Bernard Tourancheau, LIP ENS-Lyon 69364, Lyon cedex 07, France

214. Joseph F. Traub, Department of Computer Science, Columbia University, New York, NY 10027

215. Lloyd N. Trefethen, Department of Computer Science, Cornell University, Ithaca, NY 14853

216. Robert van de Geijn, University of Texas, Department of Computer Sciences, TAI 2.124, Austin, TX 78712

217. Charles Van Loan, Department of Computer Science, Cornell University, Ithaca, NY 14853

218. Udaya B. Vemulapati, Department of Computer Science, University of Central Florida, Orlando, FL 32816-0362

219. Robert G. Voigt, National Science Foundation, Room 417, 1800 G Street N.W., Washington, DC 20550

220. Bi R. Vona, Center for Numerical Analysis, RLM 13.150, University of Texas at Austin, Austin, TX 78712

221. Henk A. van der Vorst, Professor Dept. of Mathematics, Universiteit Utrecht, P.O. Box 80010, $3508 \mathrm{TA}$, Utrecht, THE NETHERLANDS

222. Michael D. Vose, 107 Ayres Hall, Department of Computer Science, University of Tennessee, Knoxville, TN 37996-1301

223. Phuong Vu, Cray Research, Inc., 19607 Franz Road, Houston, TX 77084

224. A. J. Wathen, School of Mathematics, University Walk, Bristol BSB 1TW, England

225. Robert P. Weaver, 1555 Rockmont Circle, Boulder, CO 80303

226. Mary F. Wheeler, Department of Mathematical Sciences, Rice University, P. O. Box 1892, Houston, TX 77251

227. Andrew B. White, Computing Division, Los Alamos National Laboratory, Los Alamos, NM 87545

228. John Zahorjan, Department of Computer Science and Engineering, Sieg Hall, FR35 , University of Washington, Seattle, WA 98195

229. Office of Assistant Manager for Energy Research and Development, U.S. Department of Energy, Oak Ridge Operations Office, P. O. Box 2001, Oak Ridge, TN $37831-8600$

230-231. Office of Scientific \& Technical Information, P. O. Box 62, Oak Ridge, TN 37831 
